\title{
BLOWUP FOR SYSTEMS OF CONSERVATION LAWS*
}

\author{
HELGE KRISTIAN JENSSEN ${ }^{\dagger}$
}

\begin{abstract}
We give examples of finite time blowup in sup-norm and total variation for $3 \times 3$ systems of strictly hyperbolic conservation laws. The exact solutions are explicitly constructed. In the case of sup-norm blowup we also provide an example where all other $p$-norms, $1 \leq p<\infty$, remain uniformly bounded. Finally we consider appropriate rescalings for the different types of blowup.
\end{abstract}

Key words. systems of conservation laws, blowup, total variation, sup-norm, rescaling

AMS subject classifications. 35L65, 35B05

PII. S0036141099352339

1. Introduction. We consider systems of conservation laws of the form

$$
U_{t}+F(U)_{x}=0
$$

with initial data

$$
U(x, 0)=U_{0}(x),
$$

where $U(x, t)=(u(x, t), v(x, t), w(x, t)) \in \mathbb{R}^{3}$ and $F: \mathbb{R}^{3} \rightarrow \mathbb{R}^{3}$ is smooth and strictly hyperbolic; i.e., the Jacobian $D F$ has real and distinct eigenvalues. We assume that each characteristic field is either genuinely nonlinear or linearly degenerate in the sense of Lax [21].

The existence of a weak entropy solution to the Cauchy problem for an $n \times n$ system of the form (1.1) has been established in two main cases. Either the total variation (T.V.) of the initial data is assumed to be sufficiently small, or one considers systems of two equations. In the seminal paper [15] Glimm introduced a functional consisting of a linear term giving the total variation of the solution and a quadratic term measuring the amount of waves generated by future collisions. For data close to a constant state and with small total variation the functional is decreasing in time, and a compactness argument yields a weak entropy solution to (1.1), (1.2). This solution is constructed by Glimm's scheme [15, 22] or by wave front-tracking [4, 5, 27].

Various extensions and refinements of the original result have been given. Young [33] proves a third-order estimate for wave interactions and uses this together with a reordering technique to obtain $L^{\infty}$-stability for solutions constructed by Glimm's scheme. In [31] Temple and Young derive sufficient conditions for existence of solutions to $3 \times 3$-systems with a 2-Riemann invariant when the data has small amplitude but possibly large variation. The same class of systems is considered in [32] where existence of solutions up to any prescribed time is established for data with arbitrarily large total variation and correspondingly small sup-norm. In both this work and in the work by Cheverry, a new length scale for the Cauchy problem was introduced. Using this, Cheverry [10] has showed how to relax the restriction on the variation of the initial data for $n \times n$-systems where all the fields are genuinely nonlinear. Schochet [28] shows that for $n \times n$-systems with "almost planar interactions" the conditions on the

\footnotetext{
${ }^{*}$ Received by the editors February 22, 1999; accepted for publication July 27, 1999; published electronically April 4, 2000. This work was sponsored by NorFA.

http://www.siam.org/journals/sima/31-4/35233.html

†S.I.S.S.A., Via Beirut 4, Trieste 34014, Italy (jenssen@sissa.it).
} 
initial data can be relaxed. Recently Bressan and Goatin [7] have considered Temple class systems where all fields are genuinely nonlinear. They prove the existence of an $L^{1}$-continuous semigroup for $L^{\infty}$-data with possible infinite total variation.

For the case $n=2$ stronger results have been obtained. Glimm and Lax [16] considered a large class of $2 \times 2$-systems and proved global existence of a weak solution under the much weaker assumption that the oscillation of the initial data is sufficiently small. Several works establish existence with large data for gas dynamics $[11,12,23$, $25,26]$. Similar results have been obtained by applying the theory of compensated compactness [13, 14]. Serre [30] has studied the case of $2 \times 2$ Temple class systems for which one has global existence for data with bounded variation. See [2] for $L^{1}$ continuous dependence in this case. Alber [1] has proved local existence for isentropic gas dynamics where the data have compact support and bounded variation. For an extension of this result to $n \times n$-systems, see [29]. A local uniqueness result in the case of small $B V$ perturbations of (possibly large) Riemann data was established in [6]. For existence and continuous dependence for special systems with data in $L^{\infty}$, see $[3,8]$.

Recent results on $3 \times 3$-systems by several authors [17, 18, 20, 24, 34] show that the restriction to $2 \times 2$-systems for these stronger results is essential. More specifically the authors consider the possibility of blowup in finite time of total variation or supnorm. These works present special classes and explicit examples of systems for which different types of behavior can be found. In [18] Jeffrey gave an example of blowup in finite time of the sup-norm (and, hence, also of total variation) for a $3 \times 3$-system. The system is strictly hyperbolic and linearly degenerate in each characteristic family, and the solution is smooth. A weakness of this example is that the system is not in conservative form. Young [34] constructs exact solutions to $3 \times 3$-systems with periodic initial data. Depending on the choice of initial data and the interaction coefficients one gets different types of behavior. These include arbitrarily large magnifications of total variation and $p$-norms $(1 \leq p \leq \infty)$ in finite time, decay rates like $1 /(1+t)$, exponential growth and decay, and time periodic solutions. The systems are linearly degenerate in each family (constant eigenvalues) so that the regularizing effect of genuine nonlinearity is absent and all nonlinear effects are due to the geometrical nonlinearities of the wave curves in state space. In [20] Joly, Metivier, and Rauch presented a class of systems which are genuinely nonlinear in all three fields. Using the theory of weakly nonlinear geometric optics, the authors show the existence of systems with periodic initial data where the variation grows arbitrarily large and the sup-norm is amplified by arbitrary large factors in finite time. A common feature of the works [20] and [34] is the use of initial data in $B V_{l o c}$ with sufficiently small amplitude such that the solution remains local in state space. In the former case, this guarantees that all Riemann problems can be solved uniquely, while in the latter it guarantees that the methods of weakly nonlinear geometric optics can be applied. For further work on the methods of nonlinear geometric optics applied to systems of conservation laws, see [9, 19, 24]. Recently Bressan and Shen [8] have given an example to the effect that the Cauchy problem is not well posed for $3 \times 3$-systems if one allows data with infinite total variation.

The works of Young and Joly, Metivier, and Rauch show that one cannot extend the Glimm-Lax theory to larger systems. In particular it is not possible to prove global existence for general $3 \times 3$-systems by deriving uniform $B V$-estimates. However, given these results it is still conceivable that one could obtain general global existence results for $n \times n$-systems, $n \geq 3$, even in the case of large data. We shall see that this is 


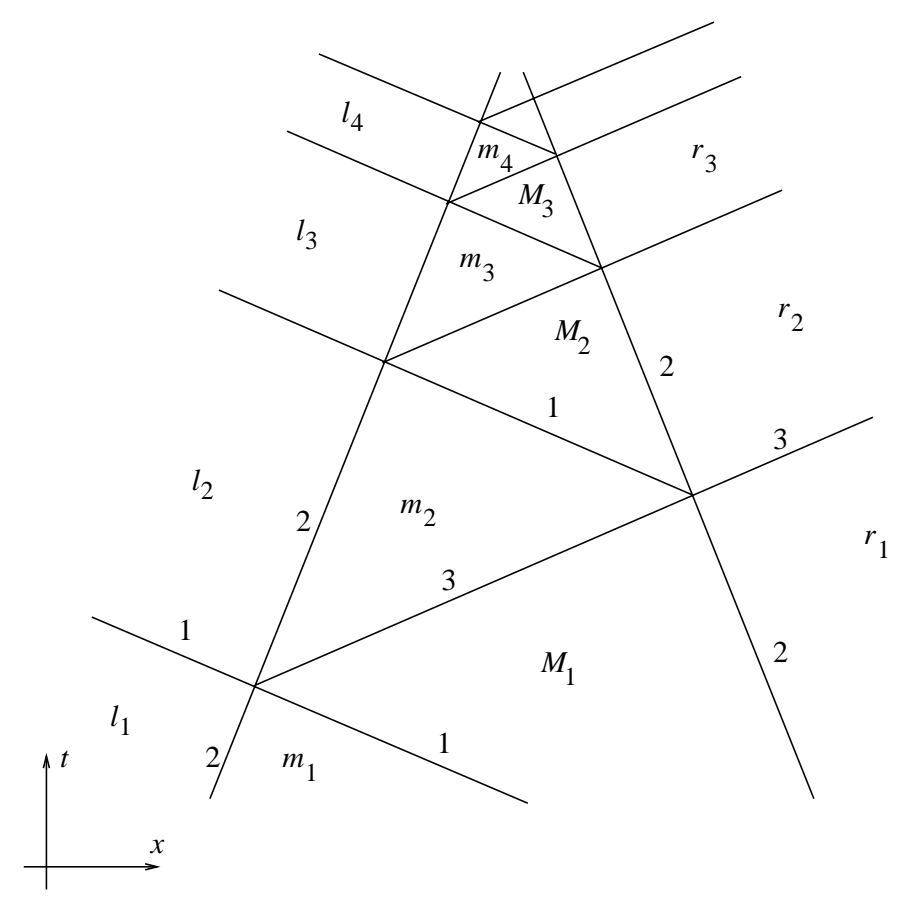

FIG. 1. Interaction pattern.

not possible. In what follows we present a class of $3 \times 3$-systems for which one can prescribe initial data such that the solution blows up in finite time. We will consider blowup in both sup-norm and total variation. That is, we give a class of examples for which there exists a time $T, 0<T<\infty$, such that

$$
\lim _{t \rightarrow T^{-}}\|U(\cdot, t)\|_{\infty}=+\infty
$$

and we also give a class of examples for which there exists a time $T, 0<T<\infty$, such that

$$
\lim _{t \rightarrow T^{-}} \text {T.V. }[U(\cdot, t)]=+\infty \quad \text { while } \quad\|U(\cdot, t)\|_{\infty} \quad \text { remains bounded. }
$$

Both types of examples are constructed by considering a situation where two 2-shocks approach each other while 1- and 3-shocks are being reflected between the 2-shocks; see Figure 1. By carefully choosing the flux function and the initial data, we obtain the above behaviors. More precisely, we get examples where the waves are magnified at each interaction, which yields blowup in sup-norm, and where the solution is periodic in state space, yielding blowup in total variation. The examples differ from the examples given in [20], [34] both in the mechanism of blowup (i.e., the particular interaction pattern) and in the fact that one actually gets infinite sup-norm or total variation in finite time. To have blowup in sup-norm the 2 -shocks must be sufficiently strong, and to have to blowup in total variation the strength of the 2-shocks must be chosen in a particular way to give periodicity in state space. However, the initial 1and 3-waves can be arbitrarily weak. We also give an example where the sup-norm blows up while the $p$-norms of $U(\cdot, t)$ remain uniformly bounded as $t \rightarrow T^{-}$. These are, to the best of my knowledge, the first examples of this type. 
The paper is organized as follows. In the next section we give the systems we will consider, we note their main properties, and we formulate the main result. In section 3 we consider Riemann problems and we derive a criteria for a Riemann problem to have a unique solution. Section 4 contains the proof of the main result. We also present rescalings which describe the asymptotics in the various cases of blowup. In the last section we collect some additional observations and comment on open problems.

2. Class of systems and statement of main result. We want to set up an interaction pattern like the one in Figure 1 where two 2-shocks approach each other while 1- and 3-waves (which will be contact discontinuities) are reflected back and forth between the 2 -shocks. Note that this requires at least three equations; i.e., it is not possible to get an interaction pattern like this for $2 \times 2$-systems. We do this by constructing solutions to $3 \times 3$-systems of the form (1.1) where the flux function $F$ has the form

$$
F(U) \equiv F(u, v, w)=\left(\begin{array}{c}
u a(v)+w \\
\Gamma(v) \\
u\left(\lambda_{0}^{2}-a^{2}(v)\right)-w a(v)
\end{array}\right) .
$$

Here $\lambda_{0}>0$ is a constant and $a(v)$ will be chosen later to obtain the types of behavior stated in Theorem 2.1. To simplify the analysis we assume that $\Gamma(v)$ has the following properties:

(i) $\Gamma(v)$ is strictly convex;

(ii) $-\lambda_{0}<\gamma(v) \equiv \Gamma^{\prime}(v)<\lambda_{0}$ for all $v \in \mathbb{R}$;

(iii) $\Gamma(0)=0$ and $\Gamma(-v)=\Gamma(v)$ for all $v \in \mathbb{R}$.

It is readily checked that the eigenvalues of the Jacobian $D F$ are

$$
\lambda_{1}=-\lambda_{0}, \quad \lambda_{2}=\gamma(v), \quad \lambda_{3}=+\lambda_{0} .
$$

Thus (ii) guarantees that the system is strictly hyperbolic. Also, since the first and third eigenvalues are constants, the 1-waves to the left of the left 2-wave and the 3 -waves to the right of the right 2-wave, respectively, do not interact (see Figure 1). The first and third eigenvectors are given by

$$
r_{1}=\left(\begin{array}{c}
1 \\
0 \\
-\left(\lambda_{0}+a(v)\right)
\end{array}\right), \quad r_{3}=\left(\begin{array}{c}
1 \\
0 \\
\lambda_{0}-a(v)
\end{array}\right) .
$$

Note that the second equation in the system is a decoupled scalar conservation law for $v$ with a strictly convex flux. It follows that the second characteristic field is genuinely nonlinear. The first and third fields are linearly degenerate so that all 1 -waves and 3-waves are contact discontinuities. It follows that shock and rarefaction curves coincide in the first and third families, and these are straight lines in planes with $v=$ constant.

Remark. This class of systems is a modification of the examples considered by Young [34]. The difference is that we have introduced nonlinearity in the second field. As in [34] we construct exact solutions.

We say that a solution of (1.1), (1.2) has an interaction pattern as in Figure 1 if the initial data consist of four constant states $U_{l_{1}}, U_{m_{1}}, U_{M_{1}}$, and $U_{r_{1}}$ (ordered from left to right) and the Riemann problem

- $\left(U_{l_{1}}, U_{m_{1}}\right)$ gives rise to a single 2 -shock with positive speed;

- $\left(U_{m_{1}}, U_{M_{1}}\right)$ gives rise to a single contact discontinuity of the first family; 
- $\left(U_{M_{1}}, U_{r_{1}}\right)$ gives rise to a single 2 -shock with negative speed.

Note that the $v$-component does not change across 1- and 3-waves. Since the second equation is a scalar conservation law for $v$ with a convex flux satisfying $\Gamma(0)=0$, it follows that the solution has an interaction pattern as in Figure 1 if and only if $0<v_{l_{1}}>v_{m_{1}}=v_{M_{1}}>v_{r_{1}}<0$. We now state the main result of the paper.

THEOREM 2.1. Let the flux $F$ be given by (2.1). Then for a suitable choice of $a(v), \Gamma(v), \lambda_{0}$, and initial states $U_{l_{1}}, U_{m_{1}}, U_{M_{1}}$, and $U_{r_{1}}$, the solution of (1.1), (1.2) has an interaction pattern as in Figure 1 and satisfies one of the following relations.

(a) There exists a time $T, 0<T<\infty$, such that

$$
\lim _{t \rightarrow T^{-}}\|U(\cdot, t)\|_{\infty}=+\infty .
$$

(b) There exist a constant $C>0$ and a time $T, 0<T<\infty$, such that

$$
\lim _{t \rightarrow T^{-}} T . V .[U(\cdot, t)]=+\infty \text {, while }\|U(\cdot, t)\|_{\infty}<C \text { for all } t<T .
$$

In the case of blowup in sup-norm one may choose the parameters so that all other $p$-norms of $U(\cdot, t), 1 \leq p<\infty$, remain uniformly bounded as $t \rightarrow T^{-}$. Moreover, in all cases the system remains uniformly strictly hyperbolic in the sense that there is a $\delta>0$ such that $-\lambda_{0}+\delta<\gamma(v(x, t))<\lambda_{0}-\delta$ for all $(x, t) \in \mathbb{R} \times[0, T)$.

3. Riemann problems. In this section we consider the Riemann problem for the system (1.1), i.e., the Cauchy problem when the data consist of two constant states,

$$
U_{0}(x)= \begin{cases}U_{l} & \text { if } x<0 \\ U_{r} & \text { if } x>0\end{cases}
$$

We will consider only the case in which $v_{l}>v_{r}$ since this is all we need to construct solutions with the properties described in the theorem. The solution of the Riemann problem then consists of a contact discontinuity of the first family connecting $U_{l}$ to some state $U^{-}$, followed by a 2 -shock connecting $U^{-}$to some state $U^{+}$, followed by a contact discontinuity of the third family connecting $U^{+}$to $U_{r}$.

We first parameterize the integral curves of the first and third family by $s$. These are straight lines and the parameterizations are readily obtained from (2.3). We let $D_{j}[s ;(\bar{u}, \bar{v}, \bar{w})]$ denote the integral curve of the $j$ th field, $j=1,3$, through the point $(\bar{u}, \bar{v}, \bar{w})$. Thus the first and third wave curves are given by

$$
D_{1}[s ;(\bar{u}, \bar{v}, \bar{w})]=\left(\begin{array}{c}
s+\bar{u} \\
\bar{v} \\
-s\left(\lambda_{0}+a(\bar{v})\right)+\bar{w}
\end{array}\right)
$$

and

$$
D_{3}[s ;(\bar{u}, \bar{v}, \bar{w})]=\left(\begin{array}{c}
s+\bar{u} \\
\bar{v} \\
s\left(\lambda_{0}-a(\bar{v})\right)+\bar{w}
\end{array}\right) .
$$

To find the expression for the 2-shock curve through $(\bar{u}, \bar{v}, \bar{w})$, we use the RankineHugoniot condition. This states that if the solution contains a discontinuity with speed $\bar{\gamma}$, then

$$
[F(U)]=\bar{\gamma}[U],
$$


where [.] denotes the jump across the discontinuity. Let the left and right states be $(\bar{u}, \bar{v}, \bar{w})$ and $(u, v, w)$, respectively. With the flux given by (2.1) the RankineHugoniot condition takes the form

$$
\begin{array}{r}
u a(v)+w-\bar{u} a(\bar{v})-\bar{w}=\bar{\gamma}(u-\bar{u}), \\
\Gamma(v)-\Gamma(\bar{v})=\bar{\gamma}(v-\bar{v}), \\
u\left(\lambda_{0}^{2}-a^{2}(v)\right)-w a(v)-\bar{u}\left(\lambda_{0}^{2}-a^{2}(\bar{v})\right)+\bar{w} a(\bar{v})=\bar{\gamma}(w-\bar{w}) .
\end{array}
$$

These relations yield three curves. Along two of these $v$ is constant and they coincide with $D_{1}$ and $D_{3}$. The third is the 2-shock curve for which we use $v$ as a parameter. Given the point $(\bar{u}, \bar{v}, \bar{w}),(3.6)$ gives the speed of the 2-shock,

$$
\bar{\gamma}=\frac{\Gamma(v)-\Gamma(\bar{v})}{v-\bar{v}} .
$$

Substituting this into (3.5) gives $w$ expressed by $u$ and $v$. Using this and (3.8) in (3.7) then yields $u$ as a function of $v$. The expressions for $u$ and $w$ are given by

$$
u=u(v ;(\bar{u}, \bar{v}, \bar{w}))=\bar{u}+\frac{a(v)-a(\bar{v})}{\lambda_{0}^{2}-\bar{\gamma}^{2}}(\bar{u}(a(\bar{v})-\bar{\gamma})+\bar{w})
$$

and

$$
w=w(v ;(\bar{u}, \bar{v}, \bar{w}))=\bar{w}+(\bar{\gamma}-a(v))[u(v ;(\bar{u}, \bar{v}, \bar{w}))-\bar{u}]-\bar{u}(a(v)-a(\bar{v})) .
$$

Note that these expressions are linear in $\bar{u}$ and $\bar{w}$.

We next use the solution of the Rankine-Hugoniot equations to derive a criteria to determine when the Riemann problem $\left(U_{l}, U_{r}\right)$ has a unique solution. Let $s_{1}$ and $s_{3}$ denote the change in parameter across the 1 -wave connecting $U_{l}$ to $U^{-}$and the 3 -wave connecting $U^{+}$to $U_{r}$, respectively. Then

$$
\begin{gathered}
U^{-}=D_{1}\left[s_{1} ; U_{l}\right], \\
U^{+}=\left(\begin{array}{c}
u\left(v_{r} ; U^{-}\right) \\
v_{r} \\
w\left(v_{r} ; U^{-}\right)
\end{array}\right),
\end{gathered}
$$

and

$$
U_{r}=D_{3}\left[s_{3} ; U^{+}\right] \text {. }
$$

This yields three equations, one for the speed of the 2-shock, given by

$$
\bar{\gamma}=\frac{\Gamma\left(v_{r}\right)-\Gamma\left(v_{l}\right)}{v_{r}-v_{l}},
$$

while the other two are linear equations for the unknown strengths $s_{1}$ and $s_{3}$. These equations can be written in the form

$$
\begin{aligned}
s_{1}\left(\bar{\gamma}+\lambda_{0}\right)+s_{3}\left(\bar{\gamma}-\lambda_{0}\right) & =A, \\
s_{1}\left[\left(\bar{\gamma}-\lambda_{0}\right)+\left(a\left(v_{r}\right)-a\left(v_{l}\right)\right)\right]+s_{3}\left(\bar{\gamma}-\lambda_{0}\right) & =B,
\end{aligned}
$$

where $A$ and $B$ are functions of $U_{l}, U_{r}$. Thus the Riemann problem $\left(U_{l}, U_{r}\right)$ has a unique solution if and only if

$$
\lambda_{0} \neq \bar{\gamma} \text {, and } a\left(v_{r}\right)-a\left(v_{l}\right)-2 \lambda_{0} \neq 0 .
$$

The first condition is always fulfilled by the mean value theorem and assumption (ii) on the flux $\Gamma$. In the proof of part (a) and (b) of Theorem 2.1 we will choose $\lambda_{0}, v_{r}$, $v_{l}$, and $a(v)$ such that the second condition is also satisfied at each interaction. 
4. Proof of main result. We now construct examples with the properties stated in Theorem 2.1. Fix a $V>0$ and let the $v$-component of the states to the left of the left 2-shock be $V$, let the $v$-component of the states between the two 2-shocks be 0 , and let the $v$-component of the states to the right of the right 2 -shock be $-V$. As noted above, this guarantees that the solution has an interaction pattern as in Figure 1. Also, since each state lies in one of the planes $v \equiv V, v \equiv 0$, or $v \equiv-V$, it is clear that the solution is uniformly strictly hyperbolic.

We next consider left interactions in which a 1-wave hits the left 2 -shock from the right. Let the states $l, m, M, l^{\prime}$, and $m^{\prime}$ be as in Figure 2. Let the strength (i.e., the change in the parameter $s$ ) of the incoming 1-wave be $S$, while the transmitted 1 -wave has strength $T$, and the reflected 3-wave has strength $R$. The given quantities are $u_{l}, v_{l}=v_{l^{\prime}}=V, w_{l}, v_{m}=v_{M}=v_{m^{\prime}}=0$, and $S$, from which we want to compute the strengths $T$ and $R$. Starting at $l$ and going either via $m$ or via $l^{\prime}$ and $m^{\prime}$ yield two expressions for the state $M$. This gives two linear equations for the strengths $T$ an $R$. We have

$$
M=D_{1}[S ; m]=D_{1}[S ;(u(0 ; l), 0, w(0 ; l))]
$$

and

$$
M=D_{3}\left[R ; m^{\prime}\right]=D_{3}\left[R ;\left(u\left(0 ; l^{\prime}\right), 0, w\left(0 ; l^{\prime}\right)\right)\right],
$$

where $l^{\prime}=D_{1}[T ; l]$. We denote the speed of the left 2 -shock by $\bar{\gamma}$, i.e.,

$$
\bar{\gamma}=\frac{\Gamma(0)-\Gamma(V)}{-V}=\frac{\Gamma(V)}{V} .
$$

We solve (4.1), (4.2) for $T$ and $R$ by using the expressions for the wave curves from above. A straightforward calculation yields

$$
T=\alpha S, \quad R=\beta S,
$$

where the magnification coefficients $\alpha$ and $\beta$ are given by

$$
\alpha=\frac{2 \lambda_{0}}{2 \lambda_{0}+a(V)-a(0)}, \quad \beta=\frac{\lambda_{0}+\bar{\gamma}}{\lambda_{0}-\bar{\gamma}}\left(\frac{a(0)-a(V)}{2 \lambda_{0}+a(V)-a(0)}\right) .
$$

Note that these coefficients depend only on $\lambda_{0}, a$, and $V$. Next consider the situation where a 3 -wave hits the right 2 -wave. Let the states $l, M, M^{\prime}, r^{\prime}$, and $r$ be as in Figure 3, and let the strength of the incoming 3 -wave, the reflected 1 -wave, and the transmitted 3 -wave be $S, R$, and $T$, respectively. The given quantities are now $u_{l}$, $v_{l}=v_{M}=v_{M^{\prime}}=0, w_{l}, v_{r}=v_{r^{\prime}}=-V$, and $S$, from which we want to compute the strengths $T$ and $R$. Starting at $l$ and going either via $M$ or via $M^{\prime}$ and $r^{\prime}$ yield two expressions for the state $r$. This gives two linear equations for the strengths $T$ and $R$. We have

$$
r=(u(-V ; M),-V, w(-V ; M)),
$$

where $M=D_{3}[S ; l]$. Also

$$
r=D_{3}\left[T ; r^{\prime}\right]=D_{3}\left[T ;\left(u\left(-V ; M^{\prime}\right),-V, w\left(-V ; M^{\prime}\right)\right)\right],
$$




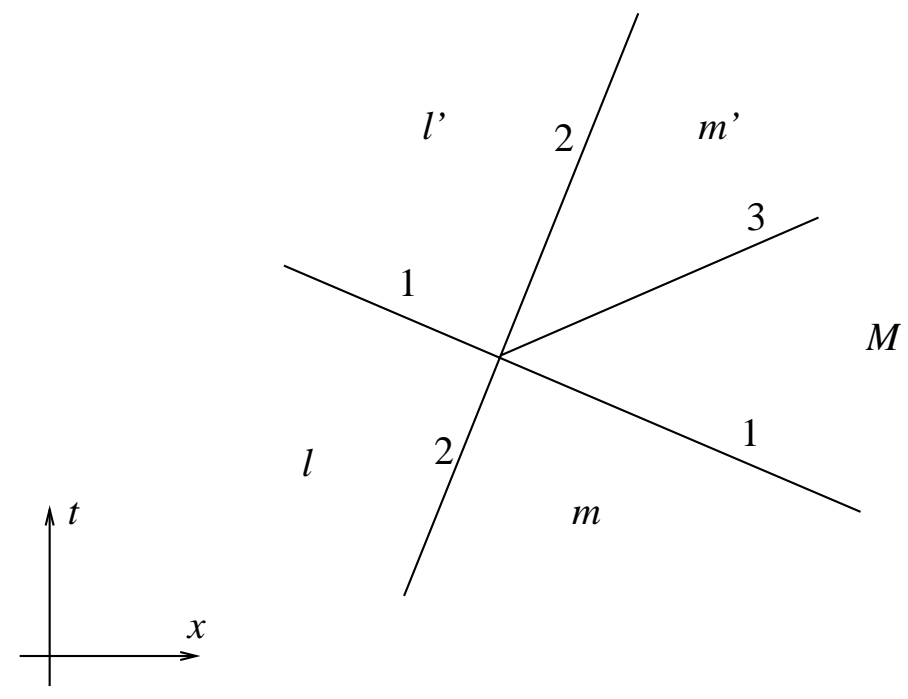

FIG. 2. Left interaction.

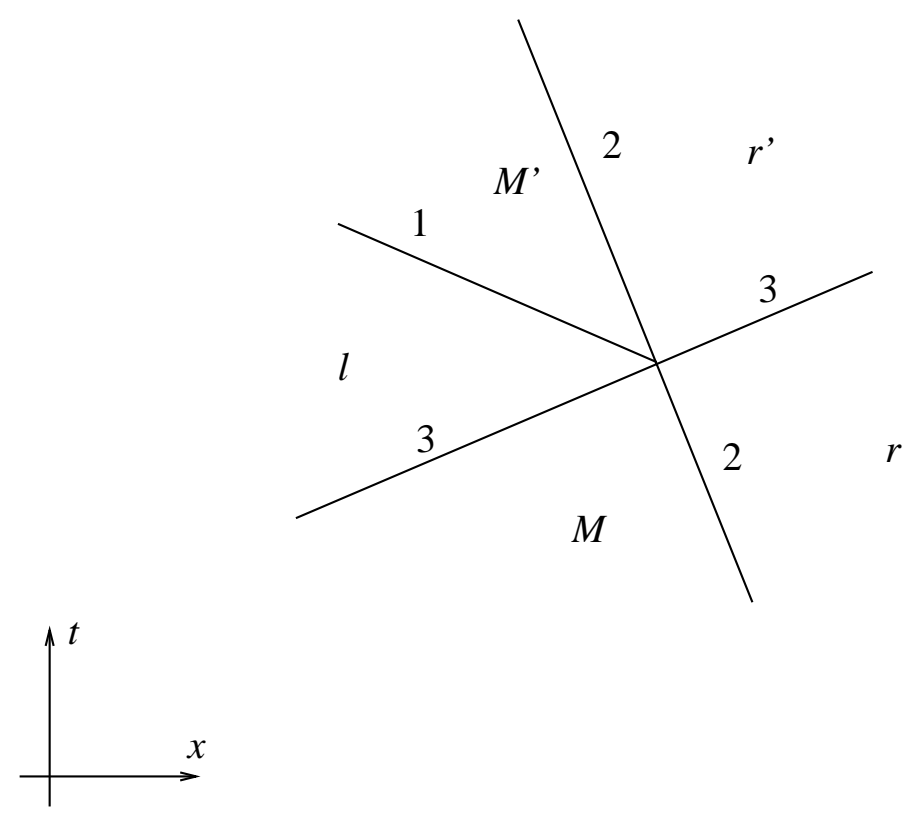

FIG. 3. Right interaction.

where $M^{\prime}=D_{1}[R ; l]$. We denote the speed of the right 2-shock by $\tilde{\gamma}$. By the properties of $\Gamma$ we have

$$
\tilde{\gamma}=\frac{\Gamma(-V)-\Gamma(0)}{-V}=-\frac{\Gamma(V)}{V}=-\bar{\gamma} .
$$

Using the expressions for the wave curves we have

$$
T=\delta S, \quad R=\varepsilon S,
$$




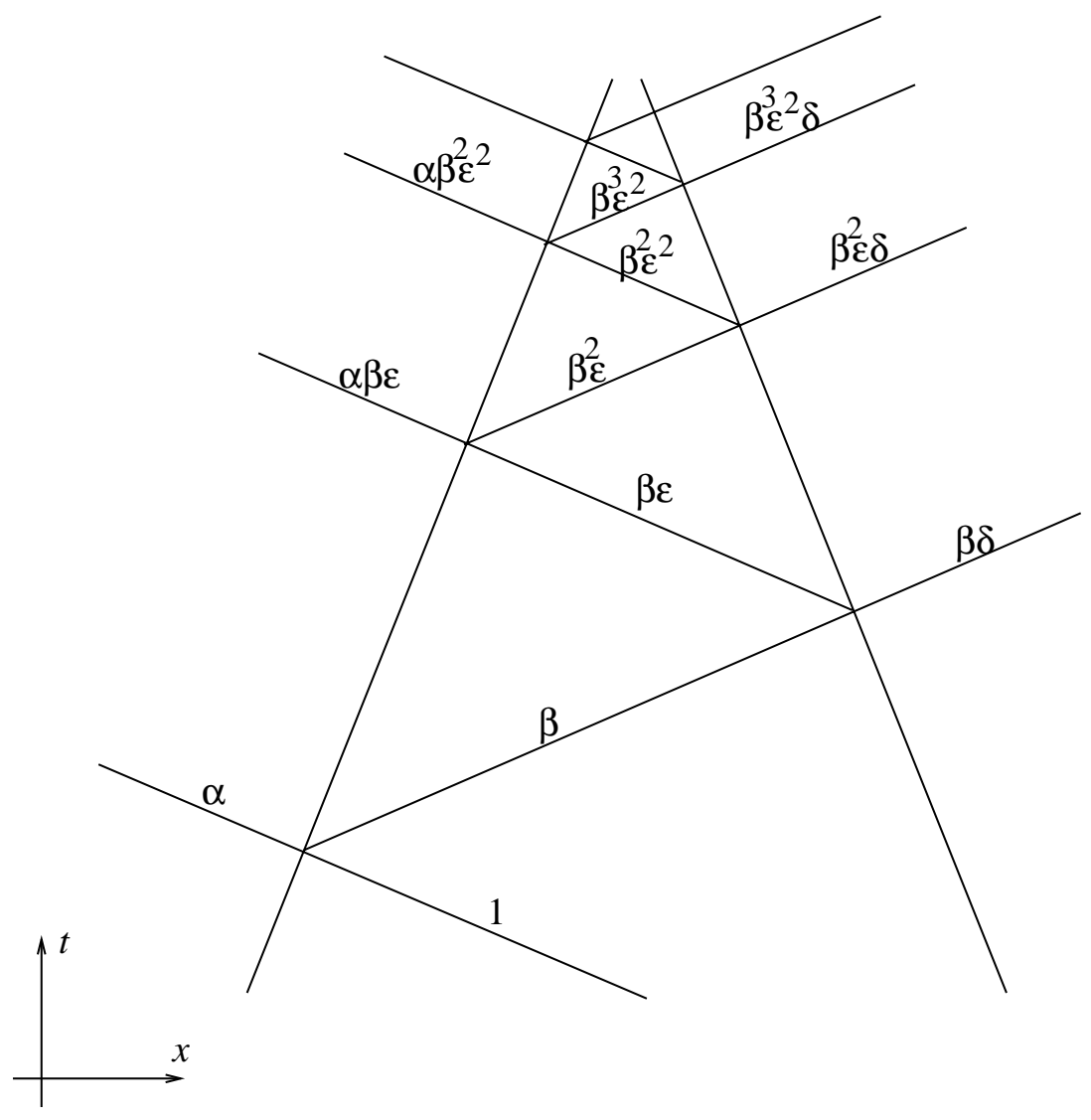

FIG. 4. Wave strengths when first incoming 1-wave has strength 1.

where the magnification coefficients $\delta$ and $\varepsilon$ are given by

$$
\delta=\frac{2 \lambda_{0}}{2 \lambda_{0}+a(0)-a(-V)}, \quad \varepsilon=\frac{\lambda_{0}+\bar{\gamma}}{\lambda_{0}-\bar{\gamma}}\left(\frac{a(-V)-a(0)}{2 \lambda_{0}+a(0)-a(-V)}\right) .
$$

As for the left interaction, the coefficients depend only on $\lambda_{0}, a$, and $V$.

Figure 4 shows the strengths of the various waves assuming that the first incoming 1-wave has strength 1.

We are now ready to choose $a, V$, and $\lambda_{0}$ so that the solution has the behavior stated in Theorem 2.1. We choose $\lambda_{0}=1$, and we let

$$
\Gamma(v)=\frac{2}{\pi} \int_{0}^{v} \arctan (\xi) d \xi,
$$

for which the properties (i)-(iii) are satisfied.

To prove part (a) we assume that $a(v)=v$. With this choice we have

$$
\alpha=\delta \text { and } \beta=\varepsilon \text {. }
$$

Also, since $V>0$, the criteria (3.17) is fulfilled, so that every Riemann problem occurring can be solved uniquely. We now refer to Figure 4 and observe that if 
$|\beta|=|\varepsilon|>1$, then the strengths of the 1- and 3-waves grow exponentially as a function of the number of interactions. Since there is an infinite number of interactions in finite time, it follows that the sup-norm tends to infinity in finite time provided $|\beta|>1$. We have

$$
|\beta|=\left(\frac{1+\bar{\gamma}}{1-\bar{\gamma}}\right)\left(\frac{V}{2+V}\right) .
$$

Since

$$
\lim _{V \rightarrow \infty} \bar{\gamma}=1,
$$

it follows that $|\beta|>1$ for $V$ large enough. This completes the proof of part (a) of Theorem 2.1. An alternative is to choose $V$ so that $\beta=\varepsilon=-1$. In this case the strengths of the transmitted 1 - and 3 -waves are constant and the sup-norm increases linearly as a function of the number of interactions taken place.

To prove part (b) we assume that $a(v)=v^{2}$. The criteria (3.17) is satisfied for the interactions along the left 2-shock, while it is satisfied for the interactions along the right 2-shock if and only if $|V| \neq \sqrt{2}$. Referring to Figures 1 and 4 , we observe that

$$
\begin{gathered}
l_{1}=l_{3}=l_{5}=\cdots, l_{2}=l_{4}=l_{6}=\cdots, \\
m_{1}=m_{3}=m_{5}=\cdots, m_{2}=m_{4}=m_{6}=\cdots, \\
M_{1}=M_{3}=M_{5}=\cdots, M_{2}=M_{4}=M_{6}=\cdots, \\
r_{1}=r_{3}=r_{5}=\cdots, r_{2}=r_{4}=r_{6}=\cdots
\end{gathered}
$$

if and only if the magnification factors $\beta$ and $\varepsilon$ satisfy

$$
\beta \varepsilon=-1 .
$$

Thus, if we can choose $V \neq \sqrt{2}$ such that $\beta \varepsilon=-1$, then the solution is periodic in state space. With $a(v)=v^{2}$ we have

$$
\beta \varepsilon=-\left(\frac{1+\bar{\gamma}}{1-\bar{\gamma}}\right)^{2} \frac{V^{4}}{4-V^{4}} .
$$

With $\Gamma(v)$ as above it is easily established that the equation $\beta \varepsilon=-1$ has a unique positive solution $V=V^{*}$. Also, $V^{*} \neq \sqrt{2}$ so that the criteria (3.17) is satisfied. We thus have a solution which is periodic in state space. Since there is an infinite number of interactions in finite time, it follows that the total variation tends to infinity in finite time while the sup-norm remains bounded. This completes the proof of part (b) of Theorem 2.1.

4.1. $\mathbf{L}^{p}$-norms. Having established the existence of solutions which blow up in either sup-norm or total variation, it is interesting to see whether one can have blowup in sup-norm while all other $p$-norms $(1 \leq p<\infty)$ of $U(\cdot, t)$ remain bounded. Of course, as $U(\cdot, t)$ takes constant nonzero values outside large enough compact intervals, this refers to the $p$-norms computed over some compact interval. We shall see that this is indeed the case where the sup-norm increases as slowly as possible. This corresponds to the case noted above where the sup-norm increases linearly as a function of the number of collisions, i.e., when $\beta=\varepsilon=-1$. We give initial data such 
that the solution has an interaction pattern as in Figure 5. That is, at time $t=0 \mathrm{a}$ 2 -shock and a 3 -wave (of strength 1 ) start at $x=-L$ and another 2 -shock starts at $x=+L$. Denote the speeds of the 2 -shocks by $\pm \bar{\gamma}$, and let $x_{n}, t_{n}$ be the coordinates of the $n$th interaction. We have

$$
x_{n}=(-1)^{n+1} b^{n} L, \quad t_{n}=\frac{L}{\bar{\gamma}}\left(1-b^{n}\right),
$$

where

$$
b=\frac{\lambda_{0}-\bar{\gamma}}{\lambda_{0}+\bar{\gamma}}
$$

Referring to Figure 5 and using the expressions for the 1- and 3-wave curves, one checks that the states in this case are given as follows:

$$
\begin{aligned}
l_{n+1} & =l_{1}-\alpha n r_{1}(+V), \\
m_{n+1} & =m_{1}+n\left[r_{3}(0)-r_{1}(0)\right], \\
M_{n+1} & =M_{1}+n\left[r_{3}(0)-r_{1}(0)\right], \\
r_{n+1} & =r_{1}+\alpha n r_{3}(-V) .
\end{aligned}
$$

Now let $t$ be a time between $t_{2 n}$ and $t_{2 n+1}$. It is readily checked that the part of $\|U(\cdot, t)\|_{p}^{p}$ corresponding to the part of the solution between the two 2-shocks is bounded by a term of the form $C b^{n} n^{p}$, while the part of $\|U(\cdot, t)\|_{p}^{p}$ corresponding to the solution to the left and right of the two 2 -shocks are bounded by sums of the form

$$
C \sum_{k=1}^{n} b^{2 k} k^{p}
$$

Since $0<b<1$, this shows that the $p$-norms are indeed bounded for all values of $p \in[1, \infty)$. This completes the proof of the theorem.

5. Rescalings and time-periodic solutions. A standard technique for studying blowup phenomena is to introduce rescaled coordinates. One seeks rescalings of both the independent and dependent variables so that the rescaled solution is nontrivial and more easily described.

Consider the type of blowup described by part (b) of Theorem 2.1. Since the solution in this case is periodic in state space, a natural question is whether one can find a rescaling of the independent variables which yields a time-periodic solution to a corresponding $3 \times 3$-system of hyperbolic equations. We will briefly describe a suitable rescaling which describes the blowup of case (b) of Theorem 2.1. We will find that the rescaled solution is periodic for large enough times on every compact interval.

Again, we give initial data such that the solution has an interaction pattern as in Figure 5 . We denote the blowup time by $T$, i.e., $T=L / \bar{\gamma}$. Denoting the rescaled time by $\tau=\tau(t)$, we want $\tau\left(t_{n+1}\right)-\tau\left(t_{n}\right)$ to be constant and equal to twice the period. The simplest way of obtaining this is to define $\tau$ by

$$
\tau=-\ln (T-t) .
$$

To have a periodic solution we must rescale the space variable such that the curves corresponding to the 2 -shocks are vertical straight lines. Denoting the new space variable by $\eta$, we rescale the $x$-variable as follows:

$$
\eta=\frac{x}{T-t} .
$$






FIG. 5. Before scaling.

The straight lines of the 2-shocks are then mapped to the two lines $\eta \equiv \pm L / T=$ $\pm \bar{\gamma}$, while the straight lines of the contact discontinuities are mapped to $\tau$-translates of exponential curves of the form

$$
\eta(\tau)= \pm\left[T\left(\lambda_{0}-\bar{\gamma}\right) e^{\tau}-\lambda_{0}\right] .
$$

The corresponding solution is then $\tau$-periodic with period $-2 \ln b$; see Figure 6 . Thus the solution of (1.1) for which we have blowup in total variation may alternatively be described as a solution of the rescaled system

$$
U_{\tau}+\eta U_{\eta}+F(U)_{\eta}=0,
$$

which is such that given any compact interval there is a time after which the solution is time-periodic on this interval.

For the case of blowup in sup-norm one has a similar result. We describe this without going into details. The scaling of the independent variables is again given by (5.1) and (5.2), while the scaling of the dependent variable is different in the two cases $|\beta|>1$ and $\beta=-1$. The new dependent variables should be

$$
\tilde{U}=\frac{U}{(T-t)^{\rho}}, \quad \tilde{U}=-\frac{U}{\ln (T-t)},
$$

respectively. Here $\rho=\ln \beta^{2} / \ln b$. The rescaled solution will tend to constant values on the $\eta$-intervals $(-\infty,-\bar{\gamma})$ and $(\bar{\gamma}, \infty)$, while it is time-periodic with period $-2 \ln b$ on the middle interval $[-\bar{\gamma}, \bar{\gamma}]$.

6. Additional observations. Consider the system (1.1) with flux (2.1) obtained by replacing $\Gamma(v)$ with

$$
\Gamma(v, k)=\frac{2}{\pi} \int_{0}^{v} \arctan (k \xi) d \xi .
$$






Fig. 6. After scaling.

By choosing large values for $k$ it is easy to show that for any $\delta>0$ one can find a system with initial data $U_{0}$ for which either

(i) $\left\|U_{0}\right\|_{\infty}<\delta$ and the solution of (1.1), (1.2) satisfies the conclusion of part (a) of Theorem 2.1

or

(ii) T.V. $\left[U_{0}\right]<\delta$ and the solution of $(1.1),(1.2)$ satisfies the conclusion of part (b) of Theorem 2.1.

However, if one has an interaction pattern as in Figure 1, then the total variation of the initial data is bounded by $6\left\|U_{0}\right\|_{\infty}$. It follows by Glimm's result that it is impossible to find a fixed system with an interaction pattern as in Figure 1 and with the property that given any $\delta>0$, there is a $U_{0}$ with $\left\|U_{0}\right\|_{\infty}<\delta$ and such that either of the behaviors in Theorem 2.1 occur.

We observe that the presence of infinitely many interactions in finite time does not necessarily imply that the solution ceases to exist. For example, in the case where $a(v)=v$, if we choose $V$ so that $|\beta|<1$, then the states to the left of the left 2-shock and the states to the right of the right 2 -shock will converge to some states $l_{\infty}$ and $r_{\infty}$, respectively. These states then define a new Riemann problem at time $t=T$ at the point where the two 2 -shocks meet. Solving this yields a 1 -wave with speed $-\lambda_{0}$, a 3 -wave with speed $+\lambda_{0}$, and a 2 -shock with an intermediate speed. All other 1- and 3 -waves from earlier interactions are prolonged and do not interact.

We observe that the type of interaction pattern as in Figure 1 is exactly what goes wrong with front-tracking for systems if one does not include a simplified Riemann solver for weak interactions. If one were to try to solve each Riemann problem in an exact manner (by solving for shocks exactly and by approximating rarefaction waves with many small shocks), then the examples above show that one may end up with infinitely many fronts in finite time. See $[3,5]$ for the definition of simplified Riemann solvers. 
The systems considered above are quite artificial. First of all it would be interesting to obtain similar results for systems where all fields are genuinely nonlinear. In this case there are additional problems due to the possible interaction of transmitted 1 - or 3 -waves. The waves created in these interactions could interact with the 2 -waves before infinitely many fronts have been created, and the analysis would be more complicated. Another basic problem is to understand the role played by entropies. Apart from the fact that the existence of a (strictly) convex entropy precludes blowup in $\mathrm{L}^{2}$, very little seems to be known.

Also note that the systems we consider are in some sense opposite to the most interesting physical example of gas dynamics. For the Euler equations, the first and third fields are genuinely nonlinear while the second field is linearly degenerate. In this case, for initial data with large total variation and correspondingly small supnorm, the results of Temple and Young [31,32] show that one cannot have more than exponential growth in total variation. The problem of whether blowup in sup-norm is possible for gas dynamics when the data have large sup-norm remains open.

Acknowledgments. I thank A. Bressan for suggesting this problem. I am indebted to B. Piccoli, C. Dafermos, H. Holden, C. Cheverry, and G.-Q. Chen for enlightening discussions. This work was completed at the Mittag-Leffler Institute, and I thank director K. O. Widman and his staff for providing excellent working conditions.

\section{REFERENCES}

[1] H.-D. Alber, Local existence of weak solutions to the quasilinear wave equation for large initial values, Math. Z., 190 (1985), pp. 249-276.

[2] P. Baiti And A. Bressan, The semigroup for Temple class system with large data, Differential Integral Equations, 10 (1997), pp. 401-418.

[3] P. Baiti and H. K. Jenssen, Well-posedness for a class of $2 \times 2$ conservation laws with $L^{\infty}$ data, J. Differential Equations, 140 (1997), pp. 161-185.

[4] P. Baiti And H. K. Jenssen, On the front tracking algorithm, J. Math. Anal. Appl., 217 (1998), pp. 395-404.

[5] A. BRessan, Global solutions to systems of conservation laws by wave-front tracking, J. Math. Anal. Appl., 170 (1992), pp. 414-432.

[6] A. Bressan And R. M. Colombo, Unique solutions of $2 \times 2$ conservation laws with large data, Indiana Univ. Math. J., 44 (1995), pp. 677-725.

[7] A. Bressan and P. Goatin, Stability of $L^{\infty}$ solutions of Temple class systems, Differential Integral Equations, to appear; also available online from http://www.math.ntnu.no/ conservation/.

[8] A. Bressan And W. Shen, Uniqueness for discontinuous O.D.E. and conservation laws, Nonlinear Anal., 34 (1998), pp. 637-652.

[9] C. Cheverry, Justification de l'optique géométrique non linéaire pour un système de lois de conservation, Duke Math. J., 87 (1997), pp. 213-263.

[10] C. Cheverry, Système de lois de conservation et stabilité BV, Mém. Soc. Math. France (N.S.), 75 (1998), pp. 1-106.

[11] R. J. DiPernA, Global solutions to a class of nonlinear hyperbolic systems of equations, Comm. Pure Appl. Math., 26 (1973), pp. 1-28.

[12] R. J. DiPernA, Existence in the large for quasilinear hyperbolic conservation laws, Arch. Rational Mech. Anal., 52 (1973), pp. 244-257.

[13] R. J. DiPerna, Convergence of approximate solutions to conservation laws, Arch. Rational Mech. Anal., 82 (1983), pp. 27-70.

[14] R. J. DiPerna, Convergence of the viscosity method for isentropic gas dynamics, Comm. Math. Phys., 91 (1983), pp. 1-30.

[15] J. Guimm, Solutions in the large for nonlinear hyperbolic systems of equations, Comm. Pure. Appl. Math., 18 (1965), pp. 697-715.

[16] J. Glimm And P. D. Lax, Decay of solutions of systems of nonlinear hyperbolic conservation laws, Mem. Amer. Math. Soc. 101, AMS, Providence, RI, 1970. 
[17] J. K. Hunter, Strongly nonlinear hyperbolic waves, in Nonlinear Hyperbolic EquationsTheory, Computation Methods, and Applications, J. Ballman and R. Jeltsch, eds., Notes Numer. Fluid Mech. 24, Vieweg, Braunschweig, 1989, pp. 257-268.

[18] A. JefFrey, Breakdown of the solution to a completely exceptional system of hyperbolic equations, J. Math. Anal. Appl., 45 (1974), pp. 375-381.

[19] J. L. Joly, G. Metivier, AND J. RAUCH, Resonant one-dimensional nonlinear geometric optics, J. Funct. Anal., 114 (1993), pp. 106-231.

[20] J. L. Joly, G. Metivier, And J. Rauch, A nonlinear instability for $3 \times 3$ systems of conservation laws, Comm. Math. Phys., 162 (1994), pp. 47-59.

[21] P. D. Lax, Hyperbolic systems of conservation laws II, Comm. Pure Appl. Math., 10 (1957), pp. 537-566.

[22] T. P. Liu, The deterministic version of the Glimm scheme, Comm. Math. Phys., 57 (1977), pp. $135-148$.

[23] T. P. LIU, Solutions in the large for the equations of nonisentropic gas dynamics, Indiana Univ. Math. J., 26 (1977), pp. 147-177.

[24] A. Majda ANd R. Rosales, Resonantly interacting weakly nonlinear hyperbolic waves. I. A single space variable, Stud. Appl. Math., 71 (1984), pp. 149-179.

[25] T. NishidA, Global solution for an initial boundary value problem of a quasilinear hyperbolic system, Proc. Japan Acad., 44 (1968), pp. 642-646.

[26] T. Nishida And J. A. Smoller, Solutions in the large for some nonlinear hyperbolic conservation laws, Comm. Pure Appl. Math., 26 (1973), pp. 183-200.

[27] N. H. Risebro, A front-tracking alternative to the random choice method, Proc. Amer. Math. Soc., 117 (1993), pp. 1125-1139.

[28] S. Schochet, Glimm's scheme for systems with almost-planar interactions, Comm. Partial Differential Equations, 16 (1991), pp. 1423-1440.

[29] S. Schochet, Sufficient conditions for local existence via Glimm's scheme for large BV data, J. Differential Equations, 89 (1991), pp. 317-354.

[30] D. SerRe, Solutions à variations bornées pour certains systèmes hyperboliques de lois de conservation, J. Differential Equations, 68 (1987), pp. 137-168.

[31] B. Temple And R. Young, Solutions to the Euler equations with large data, in Hyperbolic Problems: Theory, Numerics Applications, World Scientific, River Edge, NJ, 1996, pp. 258267.

[32] B. Temple And R. Young, The large time stability of sound waves, Comm. Math. Phys., 179 (1996), pp. 417-466.

[33] R. Young, Sup-norm stability for Glimm's scheme, Comm. Pure Appl. Math., 46 (1993), pp. 903-948.

[34] R. Young, Exact solutions to degenerate conservation laws, SIAM. J. Math. Anal., 30 (1999), pp. 537-558. 